PoS $\quad \begin{aligned} & \text { PROCEEDINGS } \\ & \text { OF SCIENCE }\end{aligned}$

\title{
NNLL resummation for QCD cross sections
}

\author{
Nikolaos Kidonakis* ${ }^{* \dagger}$ \\ Kennesaw State University, USA \\ E-mail: nkidonak@kennesaw.edu
}

I present results for the resummation of soft-gluon contributions to QCD hard-scattering cross sections at next-to-next-to-leading logarithm accuracy. A key ingredient is the calculation of twoloop soft anomalous dimensions for the partonic processes. Explicit expressions and applications are provided for processes that involve massless partons and/or massive quarks.

35th International Conference of High Energy Physics

July 22-28, 2010

Paris, France

* Speaker.

${ }^{\dagger}$ This work was supported by the National Science Foundation under Grant No. PHY 0855421. 


\section{Resummation}

QCD corrections can be quite large for many processes in hadron colliders. Certain regions of kinematical phase space can contribute the dominant part to these corrections and can be significant even beyond NLO. One such region is partonic threshold, where soft-gluon corrections are important. These corrections are needed for increased accuracy in theoretical predictions and, using factorization theorems and renormalization group evolution, they can be resummed in terms of soft anomalous dimensions. Two-loop eikonal calculations of these quantities allow the resummation to be performed at next-to-next-to-leading logarithm (NNLL) accuracy.

We resum logarithms of a moment variable $N$, conjugate to a kinematical variable that measures distance from threshold. We then write the resummed cross section as [1]

$$
\sigma(N)=\exp \left[\sum_{i} E_{i}(N)\right] H\left(\alpha_{s}\right) \exp \left[\int_{\sqrt{s}}^{\sqrt{s} / \tilde{N}} \frac{d \mu}{\mu} \Gamma_{S}^{\dagger}(\mu)\right] S\left(\frac{\sqrt{s}}{\tilde{N}}\right) \exp \left[\int_{\sqrt{s}}^{\sqrt{s} / \tilde{N}} \frac{d \mu}{\mu} \Gamma_{S}(\mu)\right]
$$

where $E_{i}$ resums universal collinear and soft-gluon emission from the incoming partons, $H$ is the hard-scattering function, $S$ is the soft-gluon function describing noncollinear soft-gluon emission, and $\Gamma_{S}$ is the soft anomalous dimension - a matrix in color space and a function of kinematical invariants. We calculate $\Gamma_{S}$ in the eikonal approximation, in momentum space and using Feynman gauge, from the UV poles of dimensionally-regularized eikonal diagrams.

\section{Two-loop soft anomalous dimensions}

Complete two-loop results for $\Gamma_{S}$ are now available for the soft (cusp) anomalous dimension for $e^{+} e^{-} \rightarrow t \bar{t}$, for $s$-channel single top production, for $b g \rightarrow t W^{-}$and $b g \rightarrow t H^{-}$, and for $t \bar{t}$ hadroproduction.

\subsection{Soft (cusp) anomalous dimension for $e^{+} e^{-} \rightarrow t \bar{t}$}

The soft (cusp) anomalous dimension for $e^{+} e^{-} \rightarrow t \bar{t}$ is $\Gamma_{S}=\left(\alpha_{S} / \pi\right) \Gamma_{S}^{(1)}+\left(\alpha_{S} / \pi\right)^{2} \Gamma_{S}^{(2)}+$ $\cdots$ with $\Gamma_{S}^{(1)}=C_{F}(\gamma \operatorname{coth} \gamma-1)$, in terms of the cusp angle $\gamma=\ln [(1+\beta) /(1-\beta)]$ with $\beta=$ $\sqrt{1-4 m^{2} / s}$ and $m$ the top quark mass, and [2]

$$
\begin{aligned}
\Gamma_{S}^{(2)}=\frac{K}{2} \Gamma_{S}^{(1)}+C_{F} C_{A}\{ & \frac{1}{2}+\frac{\zeta_{2}}{2}+\frac{\gamma^{2}}{2}-\frac{1}{2} \operatorname{coth}^{2} \gamma\left[\zeta_{3}-\zeta_{2} \gamma-\frac{\gamma^{3}}{3}-\gamma \operatorname{Li}_{2}\left(e^{-2 \gamma}\right)-\operatorname{Li}_{3}\left(e^{-2 \gamma}\right)\right] \\
& \left.-\frac{1}{2} \operatorname{coth} \gamma\left[\zeta_{2}+\zeta_{2} \gamma+\gamma^{2}+\frac{\gamma^{3}}{3}+2 \gamma \ln \left(1-e^{-2 \gamma}\right)-\operatorname{Li}_{2}\left(e^{-2 \gamma}\right)\right]\right\}
\end{aligned}
$$

where $K=C_{A}\left(67 / 18-\zeta_{2}\right)-5 n_{f} / 9$.

The cusp anomalous dimension written above is an essential component of calculations for other QCD processes, where the color structure gets more complicated with more than two colored partons involved in the process. 


\subsection{Soft anomalous dimensions for single-top production processes}

Next, we compute two-loop soft anomalous dimensions for single top production in the $s$ channel and for the associated production of a top quark with a $W$ boson or a charged Higgs.

For $s$-channel single top production the one-loop [3, 4] and two-loop [4] expressions are

$$
\begin{aligned}
& \Gamma_{S, \text { top s-ch }}^{(1)}=C_{F}\left[\ln \left(\frac{s-m^{2}}{m \sqrt{s}}\right)-\frac{1}{2}\right] \\
& \Gamma_{S, \text { top s-ch }}^{(2)}=\frac{K}{2} \Gamma_{S, \text { tops }-\mathrm{ch}}^{(1)}+C_{F} C_{A} \frac{\left(1-\zeta_{3}\right)}{4} .
\end{aligned}
$$

For associated production of a top quark with a $W^{-}, b g \rightarrow t W^{-}$, the soft anomalous dimension at one-loop [3, 5] and two-loops [5] is

$$
\begin{aligned}
& \Gamma_{S, t W^{-}}^{(1)}=C_{F}\left[\ln \left(\frac{m^{2}-t}{m \sqrt{s}}\right)-\frac{1}{2}\right]+\frac{C_{A}}{2} \ln \left(\frac{m^{2}-u}{m^{2}-t}\right) \\
& \Gamma_{S, t W^{-}}^{(2)}=\frac{K}{2} \Gamma_{S, t W^{-}}^{(1)}+C_{F} C_{A} \frac{\left(1-\zeta_{3}\right)}{4} .
\end{aligned}
$$

We find the same analytical results for the related process $b g \rightarrow t H^{-}[6,5]$.

\subsection{Soft anomalous dimension matrices for $t \bar{t}$ production}

For top-antitop production in hadron colliders, the soft anomalous dimensions were derived at one-loop in [1] and have been studied in various approaches at two-loops in $[2,7,8,9,10,11]$. For $q \bar{q} \rightarrow t \bar{t}$ the soft anomalous dimension is a $2 \times 2$ matrix while that for $g g \rightarrow t \bar{t}$ is a $3 \times 3$ matrix.

The soft anomalous dimension matrix for $q \bar{q} \rightarrow t \bar{t}$ is

$$
\Gamma_{S q \bar{q}}=\left[\begin{array}{ll}
\Gamma_{q \bar{q} 11} & \Gamma_{q \bar{q} 12} \\
\Gamma_{q \bar{q} 21} & \Gamma_{q \bar{q} 22}
\end{array}\right]
$$

At one loop, in a color basis of singlet and octet exchange in the $s$ channel, we find $[1,11]$

$$
\begin{aligned}
& \Gamma_{q \bar{q} 11}^{(1)}=-C_{F}\left[L_{\beta}+1\right], \quad \Gamma_{q \bar{q} 12}^{(1)}=\frac{C_{F}}{C_{A}} \ln \left(\frac{u_{1}}{t_{1}}\right), \quad \Gamma_{q \bar{q} 21}^{(1)}=2 \ln \left(\frac{u_{1}}{t_{1}}\right), \\
& \Gamma_{q \bar{q} 22}^{(1)}=C_{F}\left[4 \ln \left(\frac{u_{1}}{t_{1}}\right)-L_{\beta}-1\right]+\frac{C_{A}}{2}\left[-3 \ln \left(\frac{u_{1}}{t_{1}}\right)+\ln \left(\frac{t_{1} u_{1}}{s m^{2}}\right)+L_{\beta}\right],
\end{aligned}
$$

where $L_{\beta}=\left[\left(1+\beta^{2}\right) /(2 \beta)\right] \ln [(1-\beta) /(1+\beta)]$ with $\beta=\sqrt{1-4 m^{2} / s}$.

In presenting our two-loop results, we use the short-hand notation $M_{\beta}$ to denote the terms inside the curly brackets in our previous expression for the cusp anomalous dimension. Then at two loops we find [11]

$$
\begin{array}{ll}
\Gamma_{q \bar{q} 11}^{(2)}=\frac{K}{2} \Gamma_{q \bar{q} 11}^{(1)}+C_{F} C_{A} M_{\beta}, & \Gamma_{q \bar{q} 12}^{(2)}=\frac{K}{2} \Gamma_{q \bar{q} 12}^{(1)}-\frac{C_{F}}{2} N_{\beta} \ln \left(\frac{u_{1}}{t_{1}}\right), \\
\Gamma_{q \bar{q} 21}^{(2)}=\frac{K}{2} \Gamma_{q \bar{q} 21}^{(1)}+C_{A} N_{\beta} \ln \left(\frac{u_{1}}{t_{1}}\right), & \Gamma_{q \bar{q} 22}^{(2)}=\frac{K}{2} \Gamma_{q \bar{q} 22}^{(1)}+C_{A}\left(C_{F}-\frac{C_{A}}{2}\right) M_{\beta},
\end{array}
$$

with $N_{\beta}$ a subset of the terms of $M_{\beta}$. 
For the $g\left(p_{a}\right)+g\left(p_{b}\right) \rightarrow t\left(p_{1}\right)+\bar{t}\left(p_{2}\right)$ channel we choose the color basis: $c_{1}=\delta^{a b} \delta_{12}, c_{2}=$ $d^{a b c} T_{12}^{c}, c_{3}=i f^{a b c} T_{12}^{c}$. The soft anomalous dimension matrix for $g g \rightarrow t \bar{t}$ in this basis is

$$
\Gamma_{S g g}=\left[\begin{array}{ccc}
\Gamma_{g g 11} & 0 & \Gamma_{g g 13} \\
0 & \Gamma_{g g 22} & \Gamma_{g g 23} \\
\Gamma_{g g 31} & \Gamma_{g g 32} & \Gamma_{g g 22}
\end{array}\right] .
$$

At one loop we have $[1,11]$

$$
\begin{aligned}
& \Gamma_{g g 11}^{(1)}=-C_{F}\left[L_{\beta}+1\right], \quad \Gamma_{g g 22}^{(1)}=-C_{F}\left[L_{\beta}+1\right]+\frac{C_{A}}{2}\left[\ln \left(\frac{t_{1} u_{1}}{m^{2} s}\right)+L_{\beta}\right], \quad \Gamma_{g g 23}^{(1)}=\frac{C_{A}}{2} \ln \left(\frac{u_{1}}{t_{1}}\right), \\
& \Gamma_{g g 13}^{(1)}=\ln \left(\frac{u_{1}}{t_{1}}\right), \quad \Gamma_{g g 31}^{(1)}=2 \ln \left(\frac{u_{1}}{t_{1}}\right), \quad \Gamma_{g g 32}^{(1)}=\frac{N_{c}^{2}-4}{2 N_{c}} \ln \left(\frac{u_{1}}{t_{1}}\right) .
\end{aligned}
$$

At two loops we find [11]

$$
\begin{aligned}
& \Gamma_{g g 11}^{(2)}=\frac{K}{2} \Gamma_{g g 11}^{(1)}+C_{F} C_{A} M_{\beta}, \quad \Gamma_{g g 22}^{(2)}=\frac{K}{2} \Gamma_{g g 22}^{(1)}+C_{A}\left(C_{F}-\frac{C_{A}}{2}\right) M_{\beta}, \quad \Gamma_{g g 23}^{(2)}=\frac{K}{2} \Gamma_{g g 23}^{(1)}, \\
& \Gamma_{g g 13}^{(2)}=\frac{K}{2} \Gamma_{g g 13}^{(1)}-\frac{C_{A}}{2} N_{\beta} \ln \left(\frac{u_{1}}{t_{1}}\right), \quad \Gamma_{g g 31}^{(2)}=\frac{K}{2} \Gamma_{g g 31}^{(1)}+C_{A} N_{\beta} \ln \left(\frac{u_{1}}{t_{1}}\right), \quad \Gamma_{g g 32}^{(2)}=\frac{K}{2} \Gamma_{g g 32}^{(1)} .
\end{aligned}
$$

\section{References}

[1] N. Kidonakis and G. Sterman, Subleading logarithms in QCD hard scattering, Phys. Lett. B 387, 867 (1996); Resummation for QCD hard scattering, Nucl. Phys. B 505, 321 (1997) [hep-ph/9705234].

[2] N. Kidonakis, Two-loop soft anomalous dimensions and NNLL resummation for heavy quark production, Phys. Rev. Lett. 102, 232003 (2009) [arXiv:0903.2561 [hep-ph]]; Two-loop soft anomalous dimensions with massive and massless quarks, in DPF 2009 [arXiv:0910.0473 [hep-ph]].

[3] N. Kidonakis, Single top production at the Tevatron: threshold resummation and finite-order soft gluon corrections, Phys. Rev. D 74, 114012 (2006) [hep-ph/0609287].

[4] N. Kidonakis, NNLL resummation for s-channel single top quark production, Phys. Rev. D 81, 054028 (2010) [arXiv:1001.5034 [hep-ph]].

[5] N. Kidonakis, Two-loop soft anomalous dimensions for single top quark associated production with a $W^{-}$or $H^{-}$, Phys. Rev. D 82, 054018 (2010) [arXiv:1005.4451 [hep-ph]].

[6] N. Kidonakis, Charged Higgs production via $b g \rightarrow t H^{-}$at the LHC, JHEP 05, 011 (2005) [hep-ph/0412422].

[7] A. Mitov, G. Sterman, and I. Sung, Phys. Rev. D 79, 094015 (2009) [arXiv:0903.3241 [hep-ph]].

[8] T. Becher and M. Neubert, Infrared singularities of QCD amplitudes with massive partons, Phys. Rev. D 79, 125004 (2009) [arXiv:0904.1021 [hep-ph]].

[9] M. Beneke, P. Falgari, and C. Schwinn, Soft radiation in heavy-particle pair production: All-order colour structure and two-loop anomalous dimension, Nucl. Phys. B 828, 69 (2010) [arXiv:0907.1443 [hep-ph]].

[10] A. Ferroglia, M. Neubert, B.D. Pecjak, and L.L. Yang, Two-loop divergences of massive scattering amplitudes in non-abelian gauge theories, JHEP 11, 062 (2009) [arXiv:0908.3676 [hep-ph]].

[11] N. Kidonakis, Next-to-next-to-leading soft-gluon corrections for the top quark cross section and transverse momentum distribution, arXiv:1009.4935 [hep-ph]. 